\title{
Low Temperature Annealing in Low Density Polyethylene. SAXS and DSC Studies
}

\author{
Sílvio CALAFATi MoYsÉs \\ Departamento de Física, Universidade Federal do Paraná, \\ Caixa Postal 19081, 81531-990 Curitiba (PR), Brazil
}

(Received October 26, 1999)

\begin{abstract}
On the bases of small-angle X-ray scattering (SAXS) and differential scanning calorimetry (DSC) techniques it is possible to show that low density polyethylene (LDPE) annealed at $40^{\circ} \mathrm{C}$ divides its original crystal population into two: the first one melts at the annealing temperature, recrystallize and continuously thicken to improve its stability. The fraction that does not melt at $40^{\circ} \mathrm{C}$ is unable to increase its lamellar thickness, although it shows crystal lateral growth. Finally, an interesting phenomenon was detected through the SAXS analysis: a transition of polymer segments from the interphase towards to the amorphous phase. This process occurs inside the stacks of the original crystals which remain unmelted at $40^{\circ} \mathrm{C}$.

KEY WORDS Recrystallization / Annealing / Low Density Polyethylene / Small-Angle X-Ray Scattering / Differential Scanning Calorimetry /
\end{abstract}

The presence of a size distribution of non equilibrium folded chain crystals makes the crystalline polymer very susceptible to size distribution changes depending on physical conditions, such as temperature, ${ }^{1-3}$ drawing $^{4}$ or pressure. ${ }^{5}$

Different reorganization processes can occur when the crystalline polymer is thermally treated. If the applied temperature on the polymer is lower than the ones observed for the crystallization of the less stable crystal fraction the following transitional phenomena can occur: nucleation and growth of new crystals directly from the amorphous state ${ }^{6}$; lateral growth of the preexistent crystals ${ }^{1,6,7}$ and thickness growth (thickening) of the crystals., ${ }^{1,3}$ On the other hand, if the temperature is higher than the ones observed during crystallization of a particular fraction inside the crystal distribution, besides the above transitions, the melting of the fraction can occur. This new amorphous material can crystallize. The overall process is known as partial meltingrecrystallization. ${ }^{5,8}$

Most of the studies about annealing in semicrystalline polymers use temperatures not far from that of the melting of the original distribution. Therefore, at least the partial melting of the less stable fractions occurs leading to a possible ulterior recrystallization process..$^{1,2,8}$

For polymers that present a broad crystallite size distribution, as LDPE, ${ }^{9,10}$ the structural changes discussed above may happen even far from the melting temperature of the overall distribution. They can show a very unstable crystal population with very low melting temperature. Furthermore, although the crystals with higher $T_{\mathrm{m}}$ unmelt, a fraction of they can undergo crystal lateral growth and thickening.

The annealing in semicrystalline polymers usually results in more than one endothermic peak in a posterior DSC heating experiment. ${ }^{1,6,11}$ If the annealing temperature is higher, the endotherms can overlap and only the overall heat enthalpy can be calculated.111

In this work we study the possible structural changes showed by LDPE at a temperature around $70-80^{\circ} \mathrm{C}$ be- low that of its melting point. This procedure intends to avoid the generation of overlapped endotherms. By combining DSC and SAXS techniques we discuss, by one hand, the behavior of different crystal populations independently of each other. And, by the other, the quantitative changes at the level of amorphous, crystalline and diffuse transition layers. ${ }^{12,13}$

\section{EXPERIMENTAL}

Films of $100 \mu \mathrm{m}$ of unfractionated low density polyethylene $\left(M_{n}=19.075\right.$, polidispersity $\left.=5,9\right)$ were obtained by thermopressing between two copper plates at $170^{\circ} \mathrm{C}$ during $10 \mathrm{~min}$ followed by quenching in water bath at $30^{\circ} \mathrm{C}$. The films were then placed in a bidistillated water bath at $40^{\circ} \mathrm{C}$ for different time intervals, between $2 \mathrm{~min}$ and $361 \mathrm{~h}$. After this thermal treatment the sample was quickly transfered to another water bath at $30^{\circ} \mathrm{C}$.

The thermal analysis was carried out in a DSC-Du Pont 2000 equipment. All thermograms were obtained at a heating rate of $10^{\circ} \mathrm{C} \mathrm{min}{ }^{-1}$. The enthalpies under the endotherms were converted to degree of crystallinity through division by $69 \mathrm{cal} \mathrm{g}^{-1}$, the polyethylene crystal perfect enthalpy. ${ }^{14}$

The small-angle X-ray scattering experiments were performed at the SAXS station of the Laboratório Nacional de Luz Síncrotron (LNLS) ${ }^{*} .{ }^{15}$ The wavelength used was $\lambda=1.608 \AA$. The photon detection was performed by a linear position sensitive detector with 1024 channels. The acquisition time was $20 \mathrm{~min}$ for each sample. The data were treated in order to correct the parasitic scattering, the absorption and the position dependence of the efficiency of the detector. The resulting scattering curves are presented with the scattering vector $s$ $=\frac{2}{\lambda}(\sin 2 \Theta)$, where $2 \Theta$ is the scattering angle. The quantitative analysis of the SAXS data were performed through the TOPAS computer code* . 


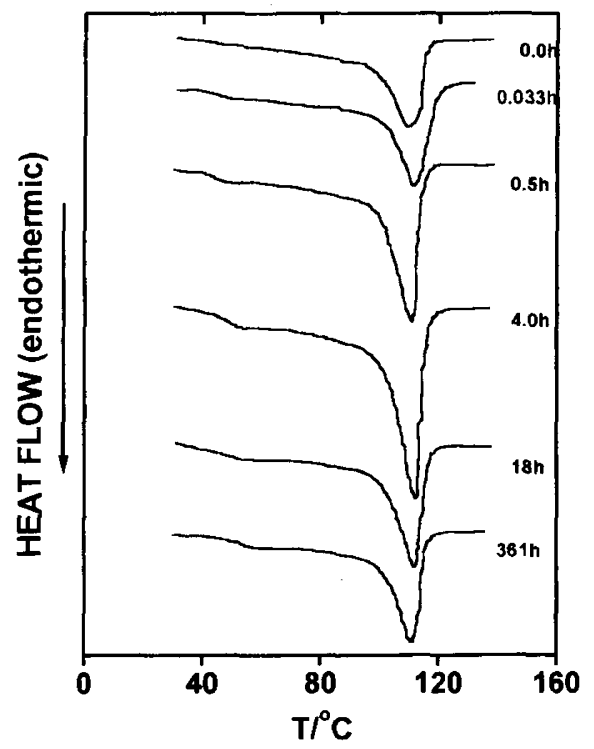

Figure 1. Thermograms evolution as a function of annealing time.

\section{RESULTS AND DISCUSSION}

\section{Differential Scanning Calorimetry}

Figure 1 shows a DSC heating thermogram for all the annealed LDPE films as well as for the unannealed one. From the thermal treatment becomes clear that with the main endotherm (I) is generated a second endotherm (II), which begins immediately above the annealing temperature $T_{\mathrm{a}}$ of $40^{\circ} \mathrm{C}$. The possibility of crystalline polymers to exhibit more than one endotherm after annealing is well known. ${ }^{1,6,16}$ In general this is obtained by using high $T_{\mathrm{a}}$ values. ${ }^{1,17}$ The results in Figure 1 show, however, that even at low annealing temperatures (about 75 ${ }^{\circ} \mathrm{C}$ below the melting temperature of the untreated film), a second endotherm arises.

This endotherm is reflecting the melting of the population of crystallites (fraction II) generated at $T_{\mathrm{a}}$ by a partial melting-recrystallization mechanism. ${ }^{5,8}$ The fraction that melts was generated during the quenching procedure, in a temperature range between 40 and $30^{\circ} \mathrm{C}$. Such fraction is formed by very thin crystallites which could be melted at $40^{\circ} \mathrm{C}$, generating amorphous material amenable to the recrystallization at this same temperature. The high temperature endotherm I reflects the melting of the crystalline fraction I with melting temperature above $40^{\circ} \mathrm{C}$.

The melting temperatures of the low temperature endothermic peak, $T_{\mathrm{m} 2}$, and the high temperature melting peak, $T_{\mathrm{m} 1}$, are displayed in Figure 2, as a function of annealing time. The last is constant, while the former increases continuously with $T_{\mathrm{a}}$. The $T_{\mathrm{m} 1}$ behavior shows that, even for long time, an annealing temperature as low as $40^{\circ} \mathrm{C}$ is unable to promote in the crystalline fraction I any effect, e.g., refolding, that would improved its thermal stability.

Since the melting point is related to the lamellar thickness ${ }^{14}$ the continuous increasing of $T_{\mathrm{m} 2}$ with the annealing time cannot be explained only by a partial melting-recrystallization mechanism. In the primary

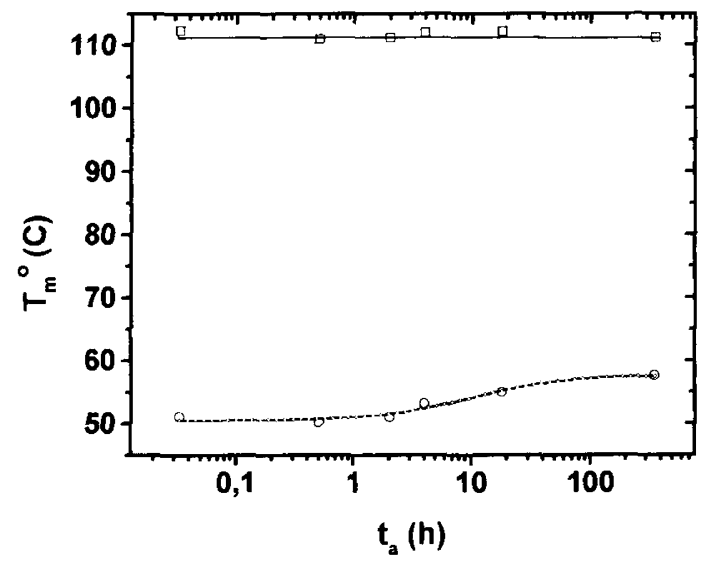

Figure 2. Time dependency of the melting temperatures of the high temperature endotherm, $T_{\mathrm{m} 1}$ (squares) and the low temperature endotherm, $T_{\mathrm{m} 2}$ (circles).

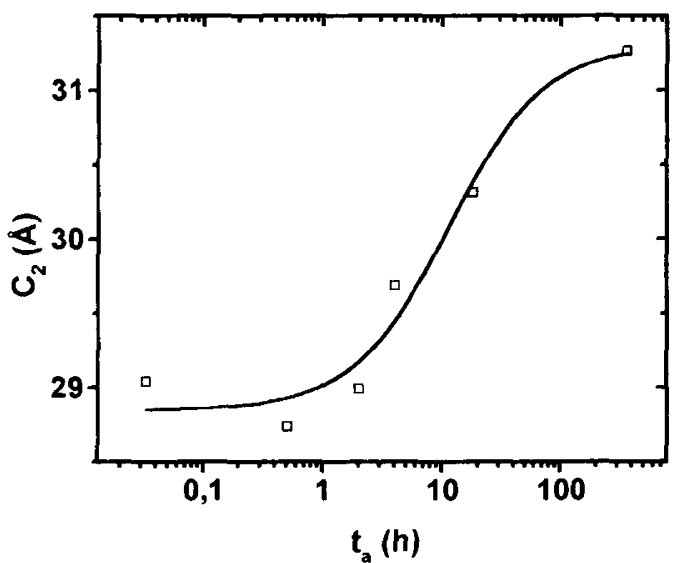

Figure 3. Time evolution of the mean lamellar thickness of the fraction II crystals.

crystallization step the final lamellar thickness value is attained at the first moments of the process. ${ }^{18}$ Therefore, the increasing of the $T_{\mathrm{m} 2}$ with the annealing time must be a consequence of some kind of thickening by solid state refolding, as it have been reported for other systems. ${ }^{3,8,17}$

The mean lamellar thickness $C_{2}$ of the crystallite fraction II can be obtained by its melting point through the Gibbs-Thomson equation, ${ }^{14}$

$$
T_{\mathrm{m} 2}=T_{\mathrm{m}}{ }^{0}\left(1-\frac{2 \sigma_{\mathrm{e}}}{C_{2} \Delta h_{\mathrm{f}}}\right)
$$

where $T_{\mathrm{m}}{ }^{0}$ is the equilibrium melting point, $\Delta h_{\mathrm{f}}$ is the perfect crystal heat of fusion by unit volume and $\sigma_{\mathrm{e}}$, the specific fold surface free energy.

The data in Figure 3 were obtained using $141.2^{\circ} \mathrm{C}$, $4.11 \mathrm{~kJ} \mathrm{~mol}^{-1} \mathrm{CH}_{2}$ and $0.0918 \mathrm{~J} \mathrm{~m}^{-2}$ for $T_{\mathrm{m}}{ }^{0}, \Delta h_{\mathrm{f}}$ and $\sigma_{\mathrm{e}}$, respectively. ${ }^{14}$ The data show that the thermal treatment at $40^{\circ} \mathrm{C}$ generates very thin crystallites, which is typical of LDPE. ${ }^{9,10}$ On the other hand, the sigmoidal shape reflects a continuous increasing of $C_{2}$ with the time, although within a short range of values. The continuous decreasing of the thickening rate $\mathrm{d} C_{2} / \mathrm{d} t$ for the longer times could be related to a greater difficulty of the 


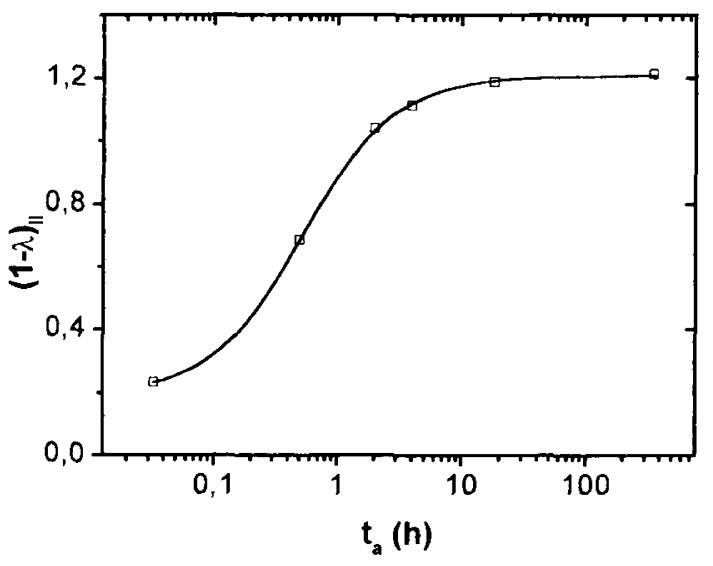

Figure 4. Time dependency of the DSC derived degree of crystallinity of the fraction II crystals.

polymer segments in the crystallite stem to translate through the crystal lattice. ${ }^{19}$ The result expressed in Figure 3 is of particular interest because the thickening is generally analized under the total crystal population and not especifically on that fraction generated at the annealing temperature.

The low temperatures region encompassed by endotherm II is a consequence of low $T_{\mathrm{a}}$ used and allows the quantitative determination of its melting enthalpy $\Delta H_{2}$ without any influence of the main endotherm. Here no endotherms overlapping is observed, even at long annealing times, differently from other cases. ${ }^{1,11}$ This permits the determination of the amount of the crystalline material generated at the annealing temperature, in an independent way of the previous crystalline fraction, unmelted at $T_{\mathrm{a}}$. Furthermore, as the heat of the endotherm II is much lower than that of the main endotherm, it may be very difficult to extract informations about the processes at the level of endotherm II through an overall heat analysis.

Figure 4 shows the degree of crystallinity $(1-\lambda)_{\text {II }}$ associated to the fraction II as a function of the annealing time. The curve is quite similar to those commonly obtained in polymer crystallization from an amorphous initial state. ${ }^{20,21}$ This may indicate that the presence of the fraction I crystals practically does not induce any change on the crystallization mechanism of the fraction II when compared to that one from amorphous initial state. As there is an inverse relation between the branching concentration and lamellar crystallite size, ${ }^{22}$ the melt of the thinnest crystals and their following crystallization must occur in a restrict spatial domain, possibly characterized by a higher branching content, within the overall branch distribution.

The total crystallinity $(1-\lambda)_{\mathrm{T}}$ related to all enthalpy under the thermogram is plotted as a function of annealing time in Figure 5. Its increasing in time is higher than the one for fraction II (Figure 4), therefore it must be connected with some changes in fraction I crystals. As the melting point of this last fraction is constant (Figure 2 ), the same occurs with its lamellar thickness. Then, the increases in crystallinity of the fraction I should be related to the lateral growth. The very rapid cooling in the quenching procedure generates at the neighborhood of the crystals unstable chain segments. They are able to

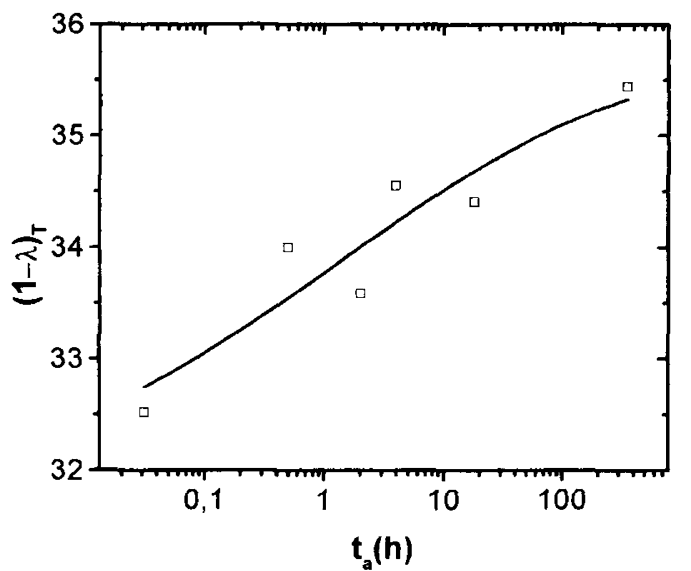

Figure 5. Time dependency of the DSC derived overall degree of crystallinity.

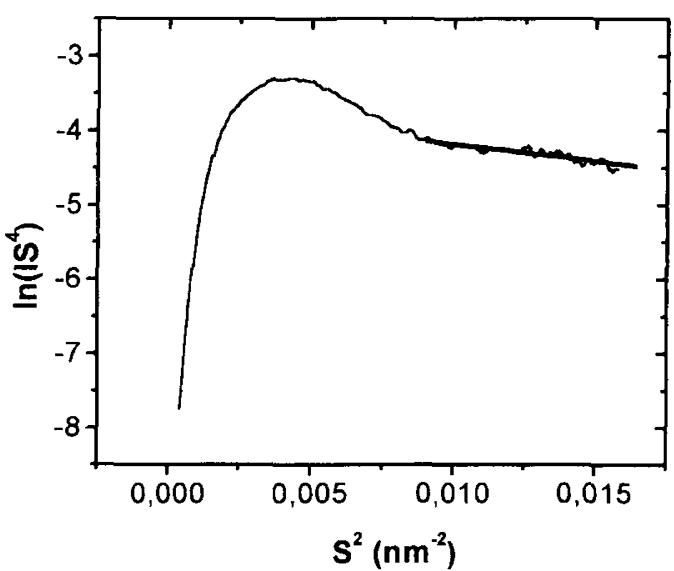

Figure 6. Modified Porod's plot to interphase determination for sample annealed during $361 \mathrm{~h}$. The straight line shows the considered region.

incorporate into the crystal even at the condition of low temperature of $40^{\circ} \mathrm{C}$.

\section{Small-Angle X-Ray Scattering}

It is known ${ }^{23}$ that the Porod's law is not followed by crystalline polymers, either by the presence of a diffuse transition layer between the crystalline and amorphous domains or by the existence of electron density fluctuations inside this domains. For these cases the scattering for the region of the large values of $s$ obey the so-called modified Porod's law ${ }^{23}$ :

$$
I_{\exp }(s)-I_{\mathrm{fl}}=\frac{K_{\mathrm{p}}}{s^{4}} \exp \left(-4 I I^{2} \sigma^{2} s^{2}\right)
$$

where the exponential is related to the Porod's deviation by the existence of the transition layer, $K_{\mathrm{p}}$ is the Porod's constant, $\sigma$ is the thickness of the transition layer and $I_{\mathrm{fl}}$ is the intensity due to electron density fluctuations within the phases. This last parameter is obtained by extrapolation from the wide-angle scattering region. Then, from a plot of $\ln \left(I_{\exp }-I_{\mathrm{fl}}\right) s^{4} v s . s^{2}$ the parameter $\sigma$ can be obtained, Figure 6 .

By multiplying the experimental intensity by the square of the scattering vector $s,{ }^{24}$ it is obtained the onedimensional Lorentz-corrected scattering curves, which 


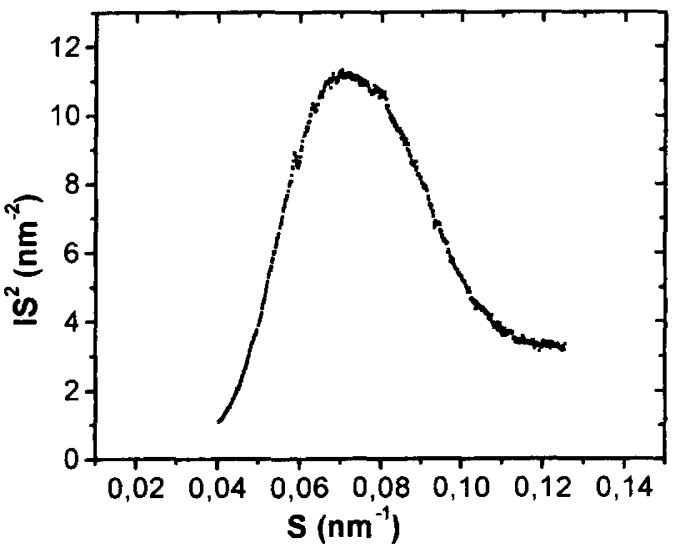

Figure 7. Lorentz-corrected scattering curve for sample annealed during $361 \mathrm{~h}$.

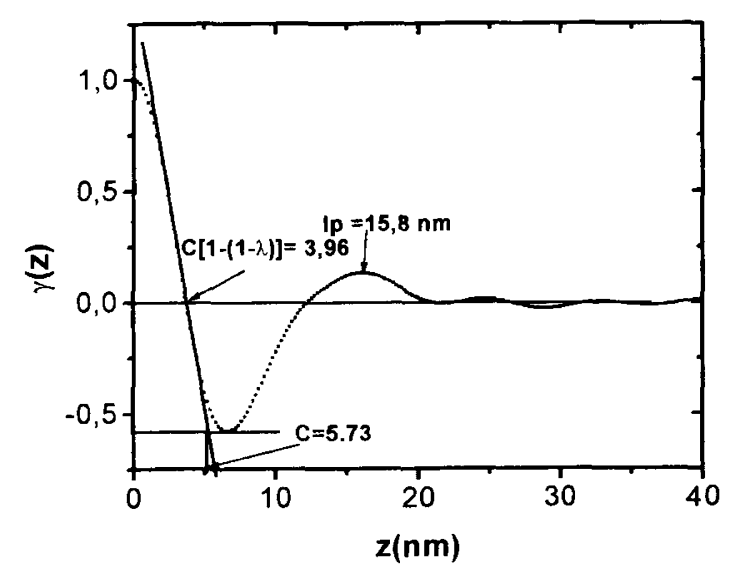

Figure 8. One-dimensional correlation function for sample annealed during $361 \mathrm{~h}$.

are shown in Figure 7.

The Fourier transformation of the Lorentz corrected scattering curve is the one-dimensional correlation function, $\gamma(z)^{24}$

$$
\gamma(z)=\int_{0}^{\infty} I_{(s)} s^{2} \cos (2 I I z s) \mathrm{d} s
$$

The $\gamma(z)$ function can be seen as a probability of finding a given electron density as a function of distance $z$ parallel to the lamellar thickness axe.

Figure 8 shows a typical result for the onedimensional correlation function. How to obtain the long period $L$, the parameter $C^{\prime}$ and the overall crystallinity volume fraction $V_{\mathrm{c}}$ are indicated in the Figure $8 .{ }^{25}$ Considering the polymer as a triphasic system, including the amorphous, crystalline and interphase, one can write ${ }^{26}$ :

$$
\begin{aligned}
& L=C+A+2 \sigma \\
& C^{\prime}=C+\sigma \\
& A^{\prime}=A+\sigma
\end{aligned}
$$

Since $\sigma$ is determined independently, both amorphous and crystalline layer thicknesses can be calculated. Figure 9 shows the time dependency of the parameters $L, C$,

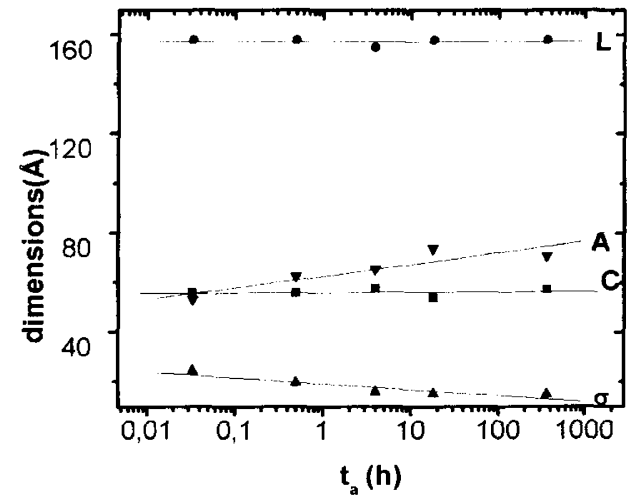

Figure 9. Effect of the annealing time on the characteristics dimensions of the LDPE.

$A$ and $\sigma$. Although from the thermal analysis it was observed some time thickening for a specific crystal population (Figure 3), there is no change in the mean lamellar thickness of the overall crystallite distribution. The invariability of the lamellar thickness of the overall crystallite distribution of the LDPE during annealing is known. . $^{27-29}$

It is important to notice that the "SAXS/correlation function value" of the mean lamellar thickness is practically the value of the fraction I crystals. On one side, it is very larger than the fraction II crystals for all annealing times as can be seen from the inspection of the Figures 4 and 5. On the other side, the scattering value of $56 \AA$, Figure 9 , is out of the range of the values obtained by eq 1 for fraction II crystals as showed in Figure 3.

On the other hand, the thermal value of the mean lamellar thickness of the fraction I crystals calculated through eq 1 is $91 \AA$, which is higher than the $56 \AA$ resulting from the SAXS/correlation function analysis (Figure 9). The difference can be attributed to a recrystallization during the DSC heating ${ }^{30}$ that generates a higher mean lamellar thickness than the original distribution. It is interesting to note that this recrystallization affects only the fraction I crystals because the crystals of the fraction II were formed at a temperature immediately below the onset of their melting. Therefore there is not any temperature range above that of the previous annealing and, at the same time, below that of the beginning of the melting.

The polymer can be thought as an infinite onedimensional stack formed continuously by the sequence of crystalline layer, interphase, amorphous layer and interphase again. ${ }^{25}$ Under this description the thicknesses of the two noncrystalline phases derived by SAXS/correlation function and showed in Figure 9 are those which form a given stack together to the fraction II crystals.

The constancy of both, lamellar thickness and long pe$\operatorname{riod}$ (Figure 9), shows that the kind of behavior of $A$ and $\sigma$ is a consequence of the continuous conversion of the diffuse transition layer to amorphous domains.

This is an interesting result, which was not reported before for LDPE. Most of the studies of annealing in LDPE shows the partial melting and the constancy ${ }^{27-29}$ or slight change ${ }^{12}$ of the lamellar thickness of the overall crystal distribution. Although some of this works have used the SAXS techniques, their data were obtained 


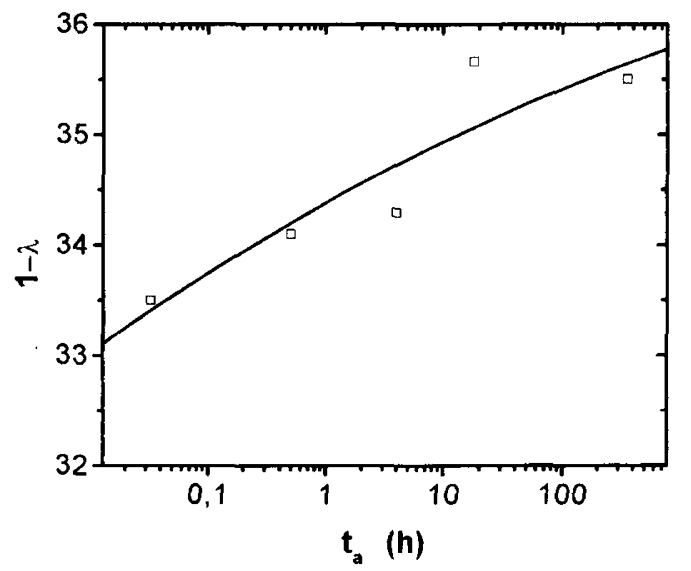

Figure 10. SAXS derived overall degree of crystallinity as a function of annealing time.

without an independent determination of the diffuse transition layer. ${ }^{12,28}$ Hence, the analysis of phases changes are limited to crystalline and noncrystalline (amorphous plus interphase).

The reasons of the transition between the two noncrystalline domains are not obvious. From a thermodynamic point of view, the transition for LDPE at $40^{\circ} \mathrm{C}$ should occur from the disordered phase to the more ordered one, since this temperature is quite distant from its thermodynamic melting point $\left(141,2^{\circ} \mathrm{C}\right) .^{14}$ However, the presence of both, high concentration and large size of branching segments, can diminish the tendency to crystallize. Therefore, those segments highly branched with very long branching could have their best thermody. namic condition as amorphous. For this reason, it is likely that this kind of segments are those ones that take place on the interphase/amorphous transition.

The overall volume fraction crystallinity $V_{c}$ is plotted as a function of time in Figure 10. As it happens with the total crystallinity derived by DSC (Figure 5), $V_{c}$ increases with time, especially at the beginning of the annealing. The numerical values of the crystallinity derived from both techniques are in quite good agreement. Following the same arguments used for the DSC discussion above, we have that the constancy of the lamellar thickness (Figure 9), implies that the crystallinity increasing is related to the crystal lateral growth, which takes place due to the incorporation of the chains or chain segments near to the crystal. The effect of this lateral growth on the overall crystallinity overcomes that of the increasing of the amorphous phase by interphase destruction, therefore the degree of crystallinity increases.

Both, the lateral growth and the unthickening show that the lateral deposition of the chains into the crystal lateral surfaces is a process which needs less energy than the translation of the polymer segments from the crystallite stem to the fold surface, a kind of solid state mechanism proposed to explain the thickening..$^{19}$ As the fraction II crystals thickens (Figure 3 ) while the mean lamellar thickness of the overall crystal distribution stays constant (Figure 9), it may be indicating that the thickening process is getting more difficult as the initial lamellar thickness value increases.

\section{CONCLUSIONS}

By suitable combination between DSC and SAXS techniques, it is possible to show that LDPE annealed at 40 ${ }^{\circ} \mathrm{C}$ exhibits three main processes. The first one, it is the melt of a very unstable crystal fraction followed by both, recrystallization and solid state lamellar thickening. The second process is related to the unmelted fraction, which, although enable to show this kind of thickening, presents lateral growth by inclusion of molecular segments, placed on the neighborhood of the crystals by the rapid cooling procedure. Finally, the third process is a transition of material from the interphase toward to amorphous region. It occurs inside those stacks that contain the crystals which remain unmelted at the annealing temperature and the long period stays unchanged. The net result of all processes is the increasing of the overall crystallinity degree with the annealing time.

Acknowledgments. The author would like to thank to Dr. Stribeck, Universitat Hamburg, Hamburg, for the TOPAS code, to LNLS for the measurements on SAXS station and to Dr. M. G. E da Luz, Universidade Federal do Paraná, Curitiba, by the manuscript revision.

\section{REFERENCES}

1. X. Lu, R. Qian, A. R. McGhie, and N. Brown, J. Polym. Sci., Phys. Ed., 30, 899 (1992).

2. Q. R. Zhu, K. L. Hong, L. Q. Ji, R. R. Qi, G. E. Zhou, M. S. Song, and Y. W. Wong, J. Polym. Sci., B: Polym. Phys., 33, 739 (1995).

3. S. J. Spells and M. J. Hill, Polymer, 32, 2716 (1991).

4. H. Freimuth, C. Sinn, and M. Dettenmaier, Polymer, 37, 831 (1996).

5. M. Yasuniwa, S. Tsubakihara, and M. Yamaguchi, J. Polym. Sci., B: Polym. Phys., 35, 535 (1997).

6. M. Huskic, J. Appl. Polym. Sci., 60, 1741 (1996).

7. K. Konnecke, Die Angewandte Makromolekulare Chemie, 198, 15 (1992).

8. E. A. Karpov, V. K. Lavrent'ev, E. Yu. Rosova, and G. K. El”yashevich, Polym. Sci., Ser. A, 37, 1247 (1995).

9. L. Mandelkern, M. Glotin, and R. A. Benson, Macromolecules, 14, 22 (1981).

10. G. R. Strobl and W. Hagedorn, J. Polym. Sci., B: Polym. Phys., 16, 1181 (1978).

11. T. Y. Ko and E. M. Woo, Polymer, 37, 1167 (1996).

12. G. R. Strobl, M. J. Schneider, and I. G. Voigt-Martin, J. Polym. Sci., B: Polym. Phys., 18, 1361 (1980).

13. H. Wang, J. M. Schultz, and B. S. Hsiao, J. Polym. Sci., B: Polym. Phys., 34, 3095 (1996).

14. B. Wunderlich and G. Czornyj, Macromolecules, 10, 906 (1977).

15. G. Kellerman, F. Vincentin, E. Tamura, M. Rocha, H. Tolentino, L. Barbosa, A. Craievich, and I. Torriani, J. Appl. Cryst., 30, 1 (1997).

16. J. Minick, A. Moet, A. Hiltner, E. Baer, and S. P. Chum, J. Appl. Polym. Sci., Polym. Phys., 58, 1371 (1995).

17. J. J. Strebel and A. Moet, J. Polym. Sci., B: Polym. Phys., 33, 1969 (1995).

18. P. J. Barham and A. Keller, J. Polym. Sci., B: Polym. Phys., 27, 1029 (1989).

19. P. J. Phillips and G. J. Rensch, J. Polym. Sci., B: Polym. Phys., 27, 155 (1989).

20. B. G. Risch, S. Srinivas, G. L. Wilkes, J. F. Geibel, C. Ash, S. White, and M. Hicks, Polymer, 37, 3623 (1996).

21. S. K. Rana, J. Appl. Polym. Sci., 61, 951 (1996).

22. Y. Kim, C. Kim, J. Park, J. Kim, and T. Min, J. Appl. Polym. 
Sci., 60, 2469 (1996).

23. W. Ruland, J. Appl. Cryst., 4, 70 (1971).

24. Y. D. Lee, P. J. Phillips, and J. S. Lin, J. Polym. Sci., B: Polym. Phys., 29, 1235 (1991).

25. M. E. Myers, A. M. Wims, T. S. Ellis, and J. Barnes, Macromolecules, 23, 2807 (1990).

26. F. Defoor, G. Groeninckx, H. Reynaers, P. Schouterden, and
B. Van der Heijden, Macromolecules, 26, 2575 (1993).

27. D. P. Pope, J. Polym. Sci., Polym. Phys., 14, 811 (1976).

28. C. G. Vonk and Y. Koga, J. Polym. Sci., Polym. Phys., 23, 2539 (1985).

29. P. J. Phillips and A. Vatansever, Polymer, 30, 710 (1989).

30. R. J. Yan and B. Jiang, J. Polym. Sci., Polym. Phys., 31, 1089 (1993). 